\title{
Expanding the Scope of C-H Amination Through Catalyst Design
}

\section{Supplementary Material \\ (14 pages)}

Christine G. Espino, Kristin Williams Fiori, Mihyong Kim, and J. Du Bois*

Department of Chemistry

Stanford University

Stanford, CA 94305-5080 
General. All reagents were obtained commercially unless otherwise noted. Reactions were performed using ovendried glassware under an atmosphere of dry nitrogen. Air- and moisture-sensitive liquids and solutions were transferred via syringe or stainless steel cannula. Organic solutions were concentrated under reduced pressure (ca. $15 \mathrm{~mm} \mathrm{Hg}$ ) by rotary evaporation. Freshly distilled solvents were employed unless otherwise noted. Dichloromethane and tetrahydrofuran were dried by passage under $12 \mathrm{psi} \mathrm{N}_{2}$ through activated alumina columns. 1,2-Dichloroethane was used as received.

Preparation and oxidative cyclization of sulfamate ester substrates followed a previously published protocol. ${ }^{1}$ The synthesis of 2,2,2-trichloroethylsulfamate was performed according to the literature report. ${ }^{2}$ Chromatographic purification of products was accomplished using forced-flow chromatography on EM Science Geduran silica gel $60(35-75 \mathrm{~mm})$. Compounds purified by chromatography on silica gel were typically applied to the adsorbent bed in dichloromethane with a minimum amount of added methanol as needed for solubility. Thin layer chromatography was performed on EM Science silica gel $60 \mathrm{~F}_{254}$ plates $(250 \mathrm{~mm})$. Visualization of the developed chromatogram was accomplished by fluorescence quenching and by staining with ethanolic anisaldehyde, aqueous potassium permanganate, or aqueous ceric ammonium molybdate (CAM) solution.

Nuclear magnetic resonance (NMR) spectra were acquired on a Varian Mercury spectrometer operating at 400 and $100 \mathrm{MHz}$ for ${ }^{1} \mathrm{H}$ and ${ }^{13} \mathrm{C}$, respectively, or on a Varian Inova spectrometer operating at 500 and $125 \mathrm{MHz}$ for ${ }^{1} \mathrm{H}$ and ${ }^{13} \mathrm{C}$, respectively, and are referenced internally according to residual solvent signals. Data for ${ }^{1} \mathrm{H}$ NMR are recorded as follows: chemical shift $(\delta, \mathrm{ppm})$, multiplicity (s, singlet; br s, broad singlet; d, doublet; br d, broad doublet; $\mathrm{t}$, triplet; q, quartet; quint, quintet; sept, septet; m, multiplet), integration, coupling constant (Hz). Data for ${ }^{13} \mathrm{C}$ NMR are reported in terms of chemical shift $(\delta, \mathrm{ppm})$. Infrared spectra were recorded on a Thermo Nicolet IR300 spectrometer and are reported in terms of frequency of absorption. Sample preparation was done either as a thin film on a $\mathrm{NaCl}$ salt plate or as a $\mathrm{KBr}$ pellet. High-resolution mass spectra were obtained from the Vincent Coates Foundation Mass Spectrometry Laboratory at Stanford University.

Experimental procedures and characterization data for $\mathbf{R h}_{2}(\mathbf{e s p})_{2}$

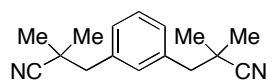

$\alpha, \alpha, \alpha^{\prime}, \alpha^{\prime}$-Tetramethyl-1,3-benzenedipropionitrile. $n$-Butyllithium (13.4 mL of a $2.5 \mathrm{M}$ solution in hexanes, 33.5 mmol, 2.5 equiv) was added slowly to a solution of $i$ - $\mathrm{Pr}_{2} \mathrm{NH}(4.7 \mathrm{~mL}, 33.5 \mathrm{mmol}, 2.5$ equiv) in $16 \mathrm{~mL}$ of THF at 0 ${ }^{\circ} \mathrm{C}$. The mixture was stirred for $20 \mathrm{~min}$ following which time isobutyronitrile $(3.0 \mathrm{~mL}, 33.5 \mathrm{mmol}, 2.5$ equiv) was added dropwise via syringe. The reaction was stirred at $0{ }^{\circ} \mathrm{C}$ for $1.5 \mathrm{~h}$. A solution of $\alpha, \alpha^{\prime}$-dichloro- $m$-xylene (2.35 $\mathrm{g}, 13.4 \mathrm{mmol}$ ) in $9 \mathrm{~mL}$ of THF was then slowly cannulated into the reaction flask. The resulting orange, slightly turbid mixture was warmed to $23{ }^{\circ} \mathrm{C}$ and stirred for $10 \mathrm{~h}$. The reaction was quenched with $50 \mathrm{~mL}$ of $\mathrm{H}_{2} \mathrm{O}$ and transferred to a separatory funnel with $150 \mathrm{~mL}$ of EtOAc. The organic layer was collected, washed with $30 \mathrm{~mL}$ of saturated aqueous $\mathrm{NaCl}$, dried over $\mathrm{MgSO}_{4}$, and concentrated under reduced pressure. Purification of the oily residue by chromatography on silica gel $(6: 1$ hexanes/EtOAc) yielded $2.4 \mathrm{~g}$ of the dinitrile product as a white solid (75\%); TLC $\mathrm{R}_{f}=0.18$ (6:1 hexanes/EtOAc): ${ }^{1} \mathrm{H}$ NMR $\left(\mathrm{CDCl}_{3}, 400 \mathrm{MHz}\right) \delta$ 7.33-7.30 (m, 1H), 7.23-7.18 (m, 3H), 2.81 (s, 4H), 1.35 (s, 12H) ppm; ${ }^{13} \mathrm{C}$ NMR $\left(\mathrm{CDCl}_{3}, 100 \mathrm{MHz}\right) \delta 135.8,132.1,129.1,128.4,124.6,46.4,33.5,26.4$ ppm; IR (thin film) $v 2979,2936,2876,2234,1608,1469,1490,1381,1369,1275,1195,807,714 \mathrm{~cm}^{-1}$.

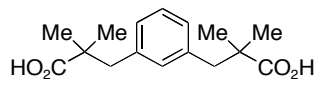

$\alpha, \boldsymbol{\alpha}, \boldsymbol{\alpha}^{\prime}, \boldsymbol{\alpha}^{\prime}$-Tetramethyl-1,3-benzenedipropionic acid. $\alpha, \alpha, \alpha^{\prime}, \alpha^{\prime}$-Tetramethyl-1,3-benzenedipropionitrile (1.1 $\mathrm{g}$, $4.58 \mathrm{mmol})$ and $\mathrm{KOH}(1.43 \mathrm{~g}, 25.4 \mathrm{mmol}, 5.6$ equiv) were combined in $6.3 \mathrm{~mL}$ of ethylene glycol. The flask was 
equipped with a Vigreux column and the mixture was heated at $180{ }^{\circ} \mathrm{C}$ for $6 \mathrm{~h}$. After cooling the reaction to $23{ }^{\circ} \mathrm{C}$, the contents were partitioned between $12 \mathrm{~mL}$ of $\mathrm{CHCl}_{3}$ and $12 \mathrm{~mL}$ of $\mathrm{H}_{2} \mathrm{O}$. The aqueous layer was collected, acidified with $8 \mathrm{~mL}$ of $6 \mathrm{M}$ aqueous $\mathrm{HCl}$, and extracted with $1 \times 90 \mathrm{~mL}$ of EtOAc. The organic layer was washed successively with $2 \times 30 \mathrm{~mL}$ of $\mathrm{H}_{2} \mathrm{O}$ and $2 \times 30 \mathrm{~mL}$ of saturated aqueous $\mathrm{NaCl}$, dried over $\mathrm{MgSO}_{4}$, and concentrated under reduced pressure. Recrystallization of the isolated material from hexanes/EtOAc afforded the desired diacid as a powdery solid $(850 \mathrm{mg}, 68 \%):{ }^{1} \mathrm{H} \mathrm{NMR}\left(\mathrm{CDCl}_{3}, 400 \mathrm{MHz}\right) \delta 7.19(\mathrm{t}, 1 \mathrm{H}, J=8.0 \mathrm{~Hz}), 7.03-7.00(\mathrm{~m}, 3 \mathrm{H}), 2.85$ (s, 4H), 1.17 (s, 12H) ppm; ${ }^{13} \mathrm{C} \mathrm{NMR}\left(\mathrm{CDCl}_{3}, 100 \mathrm{MHz}\right) \delta 184.4,137.3,131.6,128.7,127.6,45.9,43.5,24.4 \mathrm{ppm}$; IR (thin film) $v 2976,2930,2605,1698,1474,1409,1288,1218,1163,1131,944,910,734 \mathrm{~cm}^{-1}$.

$\mathbf{R h}_{2}(\mathbf{e s p})_{2}$. A variant of a protocol described by Taber and co-workers was employed. ${ }^{3}$ A $15 \mathrm{~mL}$ thick-walled tube was charged with $\mathrm{Rh}_{2}\left(\mathrm{O}_{2} \mathrm{CCF}_{3}\right)_{4}(297 \mathrm{mg}, 0.45 \mathrm{mmol}), \alpha, \alpha, \alpha^{\prime}, \alpha^{\prime}$-tetramethyl-1,3-benzenedipropionic acid (26 mg, $92 \mu \mathrm{mol}, 0.2$ equiv), and $3.0 \mathrm{~mL}$ of 1,2-dichloroethane. The vessel was sealed with a screw-cap and immersed in an oil bath preheated to $125^{\circ} \mathrm{C}$ (Note: For proper heating, the reaction flask should be immersed so that the oil level is at the height of the solvent line). At twenty minute intervals, the flask was removed from the heating bath and the contents slowly cooled $(5 \mathrm{~min})$ to $23^{\circ} \mathrm{C}$. The vessel was charged with $26 \mathrm{mg}$ ( 0.2 equiv) of the diacid and sealed, and heating at $125{ }^{\circ} \mathrm{C}$ was resumed. This process was repeated an additional three times, following which the reaction was stirred at $125^{\circ} \mathrm{C}$ for $5 \mathrm{~h}$. Within this period, the reaction mixture slowly becomes turbid. A second equivalent of dicarboxylic acid was then introduced in a similar fashion (5 portions of $26 \mathrm{mg}$ at 20 minute intervals). Once again, the mixture was stirred at $125^{\circ} \mathrm{C}$ for an additional $5 \mathrm{~h}$, during which time the reaction becomes homogenous. The deep green solution was cooled to $23{ }^{\circ} \mathrm{C}$ and the contents were applied directly to a column of silica gel $\left(150 \times 40 \mathrm{~mm}\right.$ of $\left.\mathrm{SiO}_{2}\right)$. Purification using gradient elution $(15: 1 \rightarrow 10: 1$ hexanes/EtOAc $)$ furnished $\mathrm{Rh}_{2}(\mathrm{esp})_{2}$ as a green microcrystalline product $(218 \mathrm{mg}, 64 \%)$. The first product to elute from the silica gel column using 15:1 hexanes/EtOAc is unreacted $\mathrm{Rh}_{2}(\mathrm{tfa})_{4}$ and the second is $\mathrm{Rh}_{2}(\mathrm{esp})(\mathrm{tfa})_{2}$. The third-running green band constitutes the desired complex. Solid $\mathrm{Rh}_{2}(\mathrm{esp})_{2}$ is sparingly soluble in $\mathrm{CH}_{3} \mathrm{CN}$. Crystals suitable for $\mathrm{X}$-ray diffraction were obtained by slow evaporation of 1:1 $\mathrm{CH}_{2} \mathrm{Cl}_{2} /$ acetone or $1: 1 \mathrm{CH}_{2} \mathrm{Cl}_{2} /$ acetonitrile solutions of $\mathrm{Rh}_{2}(\mathrm{esp})_{2}$. TLC $\mathrm{R}_{f}=0.43\left(20: 1 \mathrm{CH}_{2} \mathrm{Cl}_{2} / \mathrm{EtOAc}\right) ;{ }^{1} \mathrm{H} \mathrm{NMR}\left(\mathrm{CD}_{3} \mathrm{CN}, 400 \mathrm{MHz}\right) \delta 7.06(\mathrm{t}, 2 \mathrm{H}, J=7.6 \mathrm{~Hz}), 6.88(\mathrm{~s}$, $2 \mathrm{H}), 6.83(\mathrm{dd}, 4 \mathrm{H}, J=7.4,1.6 \mathrm{~Hz}), 2.58(\mathrm{~s}, 8 \mathrm{H}), 0.89(\mathrm{~s}, 24 \mathrm{H}) \mathrm{ppm}$; IR (thin film) v 3432, 1643, 1565, 1471, 1407, $1373 \mathrm{~cm}^{-1}$; HRMS $\left(\mathrm{ES}^{+}\right)$calcd for $\mathrm{C}_{32} \mathrm{H}_{40} \mathrm{O}_{8} \mathrm{Rh}_{2} 758.08$ found $781.0731\left(\mathrm{MNa}^{+}\right)$.

\section{Experimental procedure and characterization data for urea and sulfamide reactions (Figure 4)}

To a solution of substrate $(0.3 \mathrm{mmol})$ in $2.0 \mathrm{~mL}$ of toluene were added sequentially $\mathrm{MgO}(28 \mathrm{mg}, 0.69 \mathrm{mmol}, 2.3$ equiv), $\mathrm{PhI}(\mathrm{OAc})_{2}$ (106 mg, $0.33 \mathrm{mmol}, 1.1$ equiv) and $\mathrm{Rh}_{2}(\mathrm{esp})_{2}(2.3 \mathrm{mg}, 3 \mu \mathrm{mol}, 0.01$ equiv). The resulting green solution was stirred and heated at $40{ }^{\circ} \mathrm{C}$ until TLC indicated no further progress in the reaction $(\sim 10 \mathrm{~h})$. The reaction was cooled to $23{ }^{\circ} \mathrm{C}$, diluted with $4 \mathrm{~mL}$ of $\mathrm{CH}_{2} \mathrm{Cl}_{2}$, and filtered through a pad of Celite $(30 \mathrm{x} 20 \mathrm{~mm})$. The filter cake was rinsed with $2 \times 5 \mathrm{~mL}$ of $\mathrm{CH}_{2} \mathrm{Cl}_{2}$ and the combined filtrates were evaporated under reduced pressure. Purification of the isolated material by chromatography on silica gel (conditions given below) afforded the desired product.

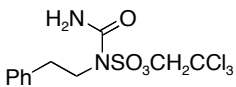

Purified by chromatography on silica gel (gradient elution: 10:1 $\rightarrow 6: 1$ hexanes/EtOAc) and recrystallized from hexanes/EtOAc; white solid (60\%): TLC $\mathrm{R}_{f}=0.4$ (3:1 hexanes/EtOAc); ${ }^{1} \mathrm{H}$ NMR $\left(\mathrm{CDCl}_{3}, 400 \mathrm{MHz}\right) \delta 7.35-7.33$ (m, 2H), 7.28-7.24 (1H, m), 7.22-7.19 (m, 2H), 7.02 (br s, 1H), 5.49 (br s, 1H), $4.63(\mathrm{~s}, 2 \mathrm{H}), 4.48$ (t, 2H, J = 6.8 Hz), 
$3.00(\mathrm{t}, 2 \mathrm{H}, J=7.2 \mathrm{~Hz}) \mathrm{ppm} ;{ }^{13} \mathrm{C} \mathrm{NMR}\left(\mathrm{CDCl}_{3}, 100 \mathrm{MHz}\right) \delta 160.4,137.0,129.1,129.0,127.2,93.9,78.7,69.7,35.0$ ppm; IR (thin film) v 3455, 3351, 1628, 1544, 1476, 1447, 1332, 1177, 1087, 1017, $853 \mathrm{~cm}^{-1}$; HRMS (ES ${ }^{-}$) calcd for $\mathrm{C}_{11} \mathrm{H}_{13} \mathrm{Cl}_{3} \mathrm{~N}_{2} \mathrm{O}_{4} \mathrm{~S} 373.9662$ found $372.9569\left(\mathrm{M}-\mathrm{H}^{+}\right)$.<smiles>O=C1NC(c2ccccc2)CN1OCCCl</smiles>

Purified by chromatography on silica gel (gradient elution: $5: 1 \rightarrow 2: 1$ hexanes/EtOAc); white solid (79\%): $\mathrm{TLC}_{f}=$ 0.25 (3:1 hexanes/EtOAc); ${ }^{1} \mathrm{H}$ NMR $\left(\mathrm{CDCl}_{3}, 400 \mathrm{MHz}\right) \delta 7.66$ (br s, $\left.1 \mathrm{H}\right), 7.47-7.41(\mathrm{~m}, 3 \mathrm{H}), 7.33-7.31(\mathrm{~m}, 2 \mathrm{H})$, $5.21(\mathrm{dd}, 1 \mathrm{H}, J=9.2,7.2 \mathrm{~Hz}), 4.94(\mathrm{t}, 1 \mathrm{H}, J=9.2 \mathrm{~Hz}), 4.73(\mathrm{~d}, 1 \mathrm{H}, J=10.8 \mathrm{~Hz}), 4.70(\mathrm{~d}, 1 \mathrm{H}, J=10.8 \mathrm{~Hz}), 4.42$ $(\mathrm{dd}, 1 \mathrm{H}, J=8.8,6.8 \mathrm{~Hz}) \mathrm{ppm} ;{ }^{13} \mathrm{C} \mathrm{NMR}\left(\mathrm{CDCl}_{3,1} 100 \mathrm{MHz}\right) \delta 163.0,137.0,130.0,129.7,126.3,93.8,78.7,74.7$, $58.9 \mathrm{ppm}$; IR (thin film) v 3384, 1628, 1480, 1460, 1440, 1342, 1177, 1101, 1017, $879 \mathrm{~cm}^{-1}$; HRMS (ES ${ }^{-}$) calcd for $\mathrm{C}_{11} \mathrm{H}_{11} \mathrm{Cl}_{3} \mathrm{~N}_{2} \mathrm{O}_{4} \mathrm{~S} 371.9505$ found $370.9411\left(\mathrm{M}-\mathrm{H}^{+}\right)$.

$$
\begin{aligned}
& \mathrm{O}_{2} \mathrm{~N}^{\text {"S' }}{ }^{\prime O} \\
& \mathrm{Me}^{-}
\end{aligned}
$$

Purified by chromatography on silica gel (gradient elution: 10:1 $\rightarrow 5: 1$ hexanes/EtOAc) and recrystallized from hexanes/EtOAc; white solid (67\%): TLC $\mathrm{R}_{f}=0.21$ (3:1 hexanes/EtOAc); ${ }^{1} \mathrm{H}$ NMR $\left(\mathrm{CDCl}_{3}, 400 \mathrm{MHz}\right) \delta 5.29$ (br s, $2 \mathrm{H}), 3.67(\mathrm{t}, 2 \mathrm{H}, J=7.2 \mathrm{~Hz}), 1.64-1.60(\mathrm{~m}, 2 \mathrm{H}), 1.53(\mathrm{~s}, 9 \mathrm{H}), 1.38-1.28(\mathrm{~m}, 2 \mathrm{H}), 0.93(\mathrm{t}, 3 \mathrm{H}, J=7.6 \mathrm{~Hz}) \mathrm{ppm} ;{ }^{13} \mathrm{C}$ NMR $\left(\mathrm{CDCl}_{3}, 100 \mathrm{MHz}\right) \delta 152.5,84.3,47.5,31.8,28.2,19.9,13.8 \mathrm{ppm}$; IR (thin film) v 3363, 3277, 2967, 2936, 2872, 1692, 1376, 1344, 1300, 1184, $1143 \mathrm{~cm}^{-1}$; HRMS (ES-) calcd for $\mathrm{C}_{9} \mathrm{H}_{20} \mathrm{~N}_{2} \mathrm{O}_{4} \mathrm{~S} 252.1144$ found 251.1062 $\left(\mathrm{M}-\mathrm{H}^{+}\right)$.

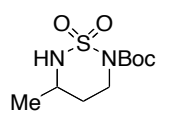

Purified by chromatography on silica gel (gradient elution: $6: 1 \rightarrow 3: 1$ hexanes/EtOAc); white solid (83\%): $\mathrm{TLC} \mathrm{R}_{f}=$ 0.15 (3:1 hexanes/EtOAc); ${ }^{1} \mathrm{H}$ NMR $\left(\mathrm{CDCl}_{3}, 400 \mathrm{MHz}\right) \delta 4.02(\mathrm{dt}, 1 \mathrm{H}, J=13.6,4.4 \mathrm{~Hz}), 3.57-3.47$ (m, $\left.1 \mathrm{H}\right), 3.40$ (ddd, $1 \mathrm{H}, J=12.0,11.2,3.2 \mathrm{~Hz}), 2.54(\mathrm{~s}, 1 \mathrm{H}), 1.76(\mathrm{dq}, 1 \mathrm{H}, J=14.0,3.6 \mathrm{~Hz}), 1.48-1.31(\mathrm{~m}, 1 \mathrm{H}), 1.36(\mathrm{~s}, 9 \mathrm{H}), 1.11$ $(\mathrm{d}, 3 \mathrm{H}, J=6.4 \mathrm{~Hz}) \mathrm{ppm} ;{ }^{13} \mathrm{C} \mathrm{NMR}\left(\mathrm{CDCl}_{3}, 100 \mathrm{MHz}\right) \delta 151.3,83.3,51.2,45.7,31.6,27.5,20.0 \mathrm{ppm}$; IR (thin film) $v$ 3254, 2980,1721, 1427, 1301, 1257, 1152, $798 \mathrm{~cm}^{-1}$; HRMS (ES ${ }^{-}$calcd for $\mathrm{C}_{9} \mathrm{H}_{18} \mathrm{~N}_{2} \mathrm{O}_{4} \mathrm{~S} 250.0987$ found $249.0897\left(\mathrm{M}-\mathrm{H}^{+}\right)$.

\section{Experimental procedure and characterization data for intermolecular $\mathbf{C}-\mathrm{H}$ amination reactions (Figure 5)}

A flask containing 2,2,2-trichloroethylsulfamate $(137 \mathrm{mg}, 0.60 \mathrm{mmol})$ in $1.2 \mathrm{~mL}$ of $\mathrm{C}_{6} \mathrm{H}_{6}$ was charged with catalyst (12 $\mu$ mol of either $\mathrm{Rh}_{2}\left(\mathrm{O}_{2} \mathrm{C}^{\mathrm{t} B u}\right)_{4}$ or $\left.\mathrm{Rh}_{2}(\mathrm{esp})_{2}\right)$ and substrate (1.0, 2.0, or 5.0 equiv). To this bright green mixture was added seven portions of $\mathrm{PhI}(\mathrm{OAc})_{2}(290 \mathrm{mg}, 0.90 \mathrm{mmol}, 1.5$ equiv) every ten minutes over the course of $1 \mathrm{~h}(7$ portions of $\sim 41 \mathrm{mg}$ ). During the addition of $\mathrm{PhI}(\mathrm{OAc})_{2}$, a change in the reaction color to brown or red was generally observed. Following the transfer of oxidant, the contents were stirred at $23{ }^{\circ} \mathrm{C}$ until TLC indicated the complete consumption of starting material or no further progress in the reaction (1-8 h). All volatiles were then removed under reduced pressure and the isolated material was purified by chromatography on silica gel (conditions given below) to afford the desired product. 


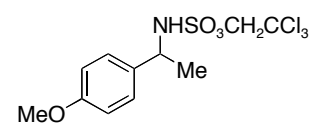

Purified by column chromatography on silica gel (9:1 hexanes/EtOAc); pale yellow oil: TLC $\mathrm{R}_{f}=0.35$ (4:1 hexanes/EtOAc); ${ }^{1} \mathrm{H}$ NMR $\left(\mathrm{CDCl}_{3}, 400 \mathrm{MHz}\right) \delta$ 7.30-7.26 (m, 2H), 6.91-6.86 (m, 2H), $5.31(\mathrm{br} \mathrm{d}, 1 \mathrm{H}, J=5.2 \mathrm{~Hz})$, 4.67 (quint, $1 \mathrm{H}, J=6.9 \mathrm{~Hz}$ ), 4.42 (d, $1 \mathrm{H}, J=10.8 \mathrm{~Hz}$ ), 4.38 (d, $1 \mathrm{H}, J=10.8 \mathrm{~Hz}), 3.79$ (s, $3 \mathrm{H}), 1.59$ (d, $3 \mathrm{H}, J=6.9$ $\mathrm{Hz}) \mathrm{ppm} ;{ }^{13} \mathrm{C} \mathrm{NMR}\left(\mathrm{CDCl}_{3}, 100 \mathrm{MHz}\right) \delta 159.3,133.5,127.5,114.2,93.3,78.0,55.3,54.3,22.7 \mathrm{ppm}$; IR (thin film) $v 3299,2978,2360,1613,1586,1515,1306,1283,1249,1178,1124,1014,977,852,833 \mathrm{~cm}^{-1}$.

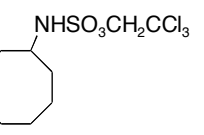

Purified by column chromatography on silica gel (gradient elution: $12: 1 \rightarrow 4: 1$ hexanes/EtOAc); white solid: TLC $\mathrm{R}_{f}$ $=0.49\left(4: 1\right.$ hexanes/EtOAc); ${ }^{1} \mathrm{H}$ NMR $\left(\mathrm{CDCl}_{3}, 400 \mathrm{MHz}\right) \delta 4.62(\mathrm{~s}, 2 \mathrm{H}), 4.54(\mathrm{br} \mathrm{d}, 1 \mathrm{H}, J=8.1 \mathrm{~Hz}), 3.74-3.65(\mathrm{~m}$, 1H), 2.04-1.96 (m, 2H), 1.74-1.64 (m, 4H), 1.63-1.48 (m, 8H) ppm; ${ }^{13} \mathrm{C}$ NMR (CDCl $\left.3,100 \mathrm{MHz}\right) \delta$ 93.5, 78.0, 55.6, 32.4, 27.1, 25.2, $23.1 \mathrm{ppm}$; IR (thin film) v 3306, 2925, 2857, 1447, 1360, 1181, 1090, 1059, 1009, 960, $855 \mathrm{~cm}^{-1}$; HRMS (ES $)$ calcd for $\mathrm{C}_{10} \mathrm{H}_{18} \mathrm{Cl}_{3} \mathrm{NO}_{3} \mathrm{~S} 359.9971$ found $359.9971\left(\mathrm{M}^{+}\right)$.

(1) Espino, C. G.; Wehn, P. M.; Chow, J.; Du Bois, J. J. Am. Chem. Soc., 2001, 123, 6935-6936.

(2) Guthikonda, K.; Du Bois, J. J. Am. Chem. Soc., 2002, 124, 13672-13673.

(3) Taber, D. F.; Meagley, R. P.; Louey, J. P.; Rheingold, A. L. Inorg. Chim. Acta, 1995, 239, 25-28. 


\title{
Crystallographic Data For $\operatorname{Rh}_{2}(\mathrm{ESP})_{2} \bullet(\text { acetone })_{2}$
}

\author{
Experimental
}

\section{$\underline{\text { Data Collection }}$}

A rod-shaped crystal of $\mathrm{C}_{38} \mathrm{H}_{52} \mathrm{O}_{10} \mathrm{Rh}_{2}$ having approximate dimensions of $0.16 \times 0.09 \times 0.09$ mm was mounted on a quartz fiber using Paratone $\mathrm{N}$ hydrocarbon oil. All measurements were made on a Siemens SMART$\mathrm{APEX}^{1} \mathrm{CCD}$ area detector with monochromatic radiation of wavelength $0.71073 \AA$.

Cell constants and an orientation matrix for data collection, obtained from a least-squares refinement using the measured positions of 2000 centered reflections with $I>10 \sigma(I)$ in the range $2.34^{\circ}<\theta<25.13^{\circ}$, corresponded to a primitive triclinic cell with dimensions:

$$
\begin{array}{ll}
a=9.731(4) \AA & \alpha=66.441(6)^{\mathrm{o}} \\
b=9.878(4) \AA & \beta=84.297(7)^{\mathrm{o}} \\
c=11.256(5) \AA & \gamma=73.066(7)^{\circ} \\
V=948.7(6) \AA^{3} &
\end{array}
$$

For $Z=1$ and $F . W .=874.62$, the calculated density is $1.531 \mathrm{~g} / \mathrm{cm}^{3}$.

Based on a statistical analysis of intensity distribution, and the successful solution and refinement of the structure, the space group was determined to be:

$\mathrm{P}-1$

The data were collected at a temperature of $159 \mathrm{~K}$. Frames corresponding to an arbitrary hemisphere of data were collected using $\omega$ scans of $0.3^{\circ}$ counted for a total of 10 seconds per frame.

\section{Data Reduction}

Data were integrated by the program SAINT $^{2}$ with box parameters of $1.6 \times 1.6 \times 1.0^{\circ}$ to a maximum $\theta$ value of $26.41^{\circ}$. The data were corrected for Lorentz and polarization effects. The linear absorption coefficient, $\mu$, for $0.71073 \AA$ radiation is $0.925 \mathrm{~mm}^{-1}$. Data were analyzed for agreement and possible absorption using SADABS ${ }^{3}$. A semi-empirical absorption correction based on 2.04 reflections with $I>5 \sigma(I)$ was applied that resulted in normalized transmission factors ranging from 0.84 to 0.92 . Of the 6182 reflections that were collected, 3767 were unique $\left(R_{\text {int }}=0.0183\right)$; equivalent reflections were merged. No decay correction was deemed necessary.

\section{$\underline{\text { Structure Solution and Refinement }}$}

The structure was solved by direct methods (SIR-92) ${ }^{4}$ and expanded using Fourier techniques ${ }^{5}$. All non-H atoms were refined with anisotropic thermal parameters. Hydrogen atoms were located using the HFIX command (idealized positions via a riding refinement). A single torsional parameter about the $\mathrm{H}-\mathrm{C}$ bond was refined in the case of methyl groups. The final cycle of full-matrix least-squares refinement ${ }^{6}$ was based on 3767 reflections (all data) and 232 variable parameters and converged (largest parameter shift was 0.002 times its esd) with conventional unweighted and weighted agreement factors of:

$$
\begin{gathered}
R_{1}=\Sigma\left\|F_{o}|-| F_{c}\right\| / \Sigma\left|F_{o}\right|=0.0268 \text { for } 3031 \text { data with } F_{o}>4 \sigma\left(F_{o}\right) \\
\mathrm{w} R_{2}=\left[\left(\Sigma w\left(\left|F_{o}\right|^{2}-\left|F_{c}\right|^{2}\right)^{2} / \Sigma w\left|F_{o}\right|^{2}\right)\right]^{1 / 2}=0.0567
\end{gathered}
$$


The standard deviation of an observation of unit weight $(S)^{7}$ was 0.947 . Sheldrick weights ${ }^{6}$ were used; where applicable, weights were refined to convergence. The maximum and minimum peaks on the final difference Fourier map corresponded to 0.57 and $-0.30 \mathrm{e}^{-} / \AA^{3}$, respectively.

Neutral atom scattering factors were taken from Cromer and Waber ${ }^{8}$. Anomalous dispersion effects were included in Fcalc'; the values for $\Delta f^{\prime}$ and $\Delta f^{\prime \prime}$ were those of Creagh and McAuley ${ }^{10}$. The values for the mass attenuation coefficients were those of Creagh and Hubbel ${ }^{11}$. All calculations were performed using the Crystal Structure ${ }^{12}$ crystallographic software package.

\section{References}

(1) SMART: Area-Detector Software Package, Siemens Industrial Automation, Inc.: Madison, WI (1995).

(2) SAINT: SAX Area-Dectector Integration Program, V5.04; Siemens Industrial Automation, Inc.: Madison, WI, (1995)

(3) SADABS: Siemens Area Detector ABSorption correction program, George Sheldrick, (1996). Advance copy, private communication.

(4) SIR92: a program for automatic solution of crystal structures by direct methods. Altomare, A., Cascarano, G., Giacovazzo G., Guagliardi A., Burla M.C., Polidori, G. \& Camalli, M. J. Appl. Cryst. (27), 435 (1994).

(5) DIRDIF99: Beurskens, P.T., Admiraal, G., Beurskens, G., Bosman, W.P., de Gelder, R., Israel, R. and Smits, J.M.M. (1999). The DIRDIF-99 program system, Technical Report of the Crystallography Laboratory, University of Nijmegen, The Netherlands.

(6) Least-Squares:

Function minimized: $\Sigma w\left(\left|F_{o}\right|^{2}-\left|F_{c}\right|^{2}\right)^{2}$

$w=1 /\left[\sigma^{2}\left(F_{o}^{2}\right)+(0.0292 \mathrm{P})^{2}\right]$ where $\mathrm{P}=\left(F_{o}^{2}+2 \mathrm{~F}_{\mathrm{c}}^{2}\right) / 3$

Sheldrick weights: G. M. Sheldrick (1997)

(7) Standard deviation of an observation of unit weight:

$$
\begin{array}{cl}
S=[\Sigma w & \left.\left(\left|F_{o}\right|^{2}-\left|F_{c}\right|^{2}\right)^{2} /\left(N_{\mathrm{o}}-N_{\mathrm{v}}\right)\right]^{1 / 2} \\
\text { where: } & N_{\mathrm{o}}=\text { number of observations } \\
& N_{\mathrm{v}}=\text { number of variables }
\end{array}
$$

(8) Cromer, D. T. \& Waber, J. T.; "International Tables for X-ray Crystallography", Vol. IV, The Kynoch Press, Birmingham, England, Table 2.2 A (1974).

(9) Ibers, J. A. \& Hamilton, W. C.; Acta Crystallogr., 17, 781 (1964).

(10) Creagh, D. C. \& McAuley, W.J .; "International Tables for Crystallography", Vol C, (A.J.C. Wilson, ed.), Kluwer Academic Publishers, Boston, Table 4.2.6.8, pages 219-222 (1992).

(11) Creagh, D. C. \& Hubbell, J.H..; "International Tables for Crystallography", Vol C, (A.J.C. Wilson, ed.), Kluwer Academic Publishers, Boston, Table 4.2.4.3, pages 200-206 (1992).

(12) CrystalStructure 3.51: Crystal Structure Analysis Package, Rigaku and MSC (2000-3).

(13) CRYSTALS Issue 10: Watkin, D.J.; Prout, C.K.; Carruthers, J.R.; Betteridge, P.W. Chemical Crystallography Laboratory, Oxford, UK. 


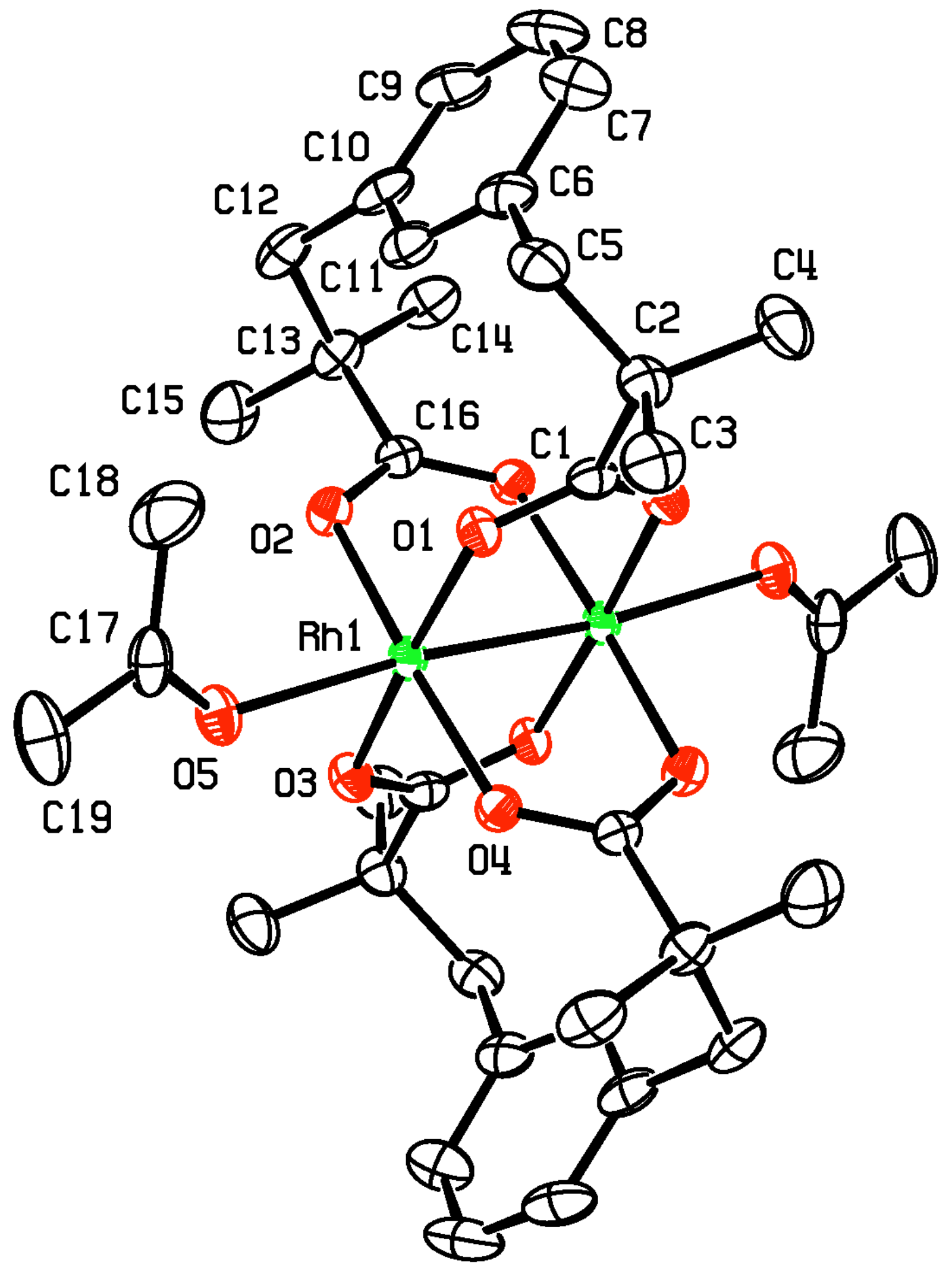




\section{Experimental Details}

A. Crystal Data

Empirical Formula

Formula Weight

Crystal Color, Habit

Crystal Dimensions

Crystal System

Lattice Type

Lattice Parameters

Space Group

$Z$ value

$d_{\text {calc }}$

$F_{000}$

$\mu(0.71073 \AA ̊$ radiation $)$

\section{B. Intensity Measurements}

Diffractometer

Radiation

Exposure Time

Scan Type

$\theta_{\max }$

Data Collection Temperature

No. of Reflections Measured

Corrections

\section{Structure Solution and Refinement}

Structure Solution

Refinement

Function Minimized

Least Squares Weighting scheme

Anomalous Dispersion

No. Observations $\left(F_{o}>4 \sigma\left(F_{o}\right)\right)$

No. Variables

Reflection/Parameter Ratio

Residuals: $R_{1}$; w $R_{2}$

Goodness of Fit Indicator $(S)$

Max Shift/Error in Final Cycle

Maximum peak in Final Diff. Map

Minimum peak in Final Diff. Map

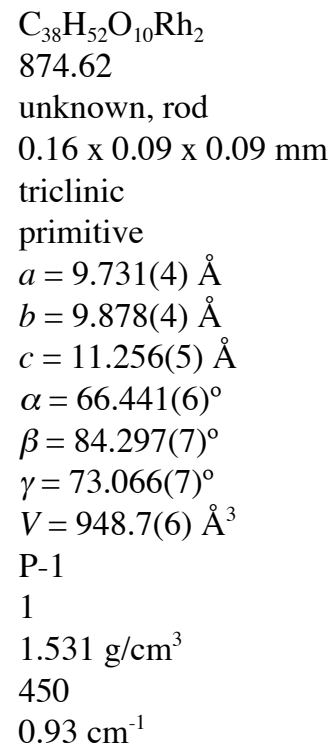

Siemens SMART-APEX CCD

$\lambda=0.71073 \AA$

graphite monochromated

10 seconds per frame.

$\omega$ (0.3 degrees per frame)

$26.41^{\circ}$

$159 \mathrm{~K}$

Total: 6182

Unique: $3767\left(R_{\mathrm{int}}=0.0183\right)$

Lorentz-polarization

Absorption:

$T_{\text {max }}=0.92$

$T_{\min }=0.84$

Direct (SIR-92)

Full-matrix least-squares (SHELXL-97)

$\Sigma w\left(\left|F_{o}\right|^{2}-\left|F_{c}\right|^{2}\right)^{2}$

$w=1 /\left[\sigma^{2}\left(F_{o}^{2}\right)+(0.0292 \mathrm{P})^{2}\right]$ where $\mathrm{P}=\left(F_{o}^{2}+2 \mathrm{~F}_{\mathrm{c}}{ }^{2}\right) / 3$

All non-hydrogen atoms

3031

232

16.24

$0.0268 ; 0.0567$

0.947

0.002

$0.57 \mathrm{e}^{-} / \AA^{3}$

$-0.30 \mathrm{e}^{-} / \AA^{3}$ 
Table 1a. Atomic coordinates, $U_{\text {iso }} / U_{\text {eq }}$, and occupancy

\begin{tabular}{|c|c|c|c|c|c|}
\hline atom & $x$ & $y$ & $z$ & $U_{\mathrm{eq}}$ & occ \\
\hline Rh1 & $0.0302(1)$ & $0.1029(1)$ & 1.0111(1) & $0.017(1)$ & 1 \\
\hline $\mathrm{O} 1$ & $0.2035(2)$ & $0.0663(2)$ & $0.8986(2)$ & $0.021(1)$ & 1 \\
\hline $\mathrm{O} 2$ & $-0.0977(2)$ & $0.2492(2)$ & $0.8513(2)$ & $0.022(1)$ & 1 \\
\hline $\mathrm{O} 3$ & $-0.1451(2)$ & $0.1270(2)$ & $1.1238(2)$ & $0.022(1)$ & 1 \\
\hline $\mathrm{O} 4$ & $0.1523(2)$ & $-0.0528(2)$ & 1.1693(2) & $0.021(1)$ & 1 \\
\hline $\mathrm{O} 5$ & $0.0934(2)$ & $0.2865(2)$ & $1.0540(2)$ & $0.027(1)$ & 1 \\
\hline $\mathrm{C} 1$ & $0.2241(3)$ & $-0.0390(3)$ & $0.8558(2)$ & $0.021(1)$ & 1 \\
\hline $\mathrm{C} 2$ & $0.3586(3)$ & $-0.0605(3)$ & $0.7753(3)$ & $0.026(1)$ & 1 \\
\hline C3 & $0.4888(3)$ & $-0.0955(3)$ & $0.8600(3)$ & $0.033(1)$ & 1 \\
\hline $\mathrm{C} 4$ & $0.3737(3)$ & $-0.1921(3)$ & $0.7316(3)$ & $0.037(1)$ & 1 \\
\hline C5 & $0.3497(3)$ & $0.0929(3)$ & $0.6558(3)$ & $0.029(1)$ & 1 \\
\hline C6 & $0.2152(3)$ & $0.1557(3)$ & $0.5725(3)$ & $0.029(1)$ & 1 \\
\hline C7 & $0.2095(3)$ & $0.1212(4)$ & $0.4649(3)$ & $0.037(1)$ & 1 \\
\hline $\mathrm{C} 8$ & $0.0824(4)$ & $0.1737(4)$ & $0.3957(3)$ & $0.046(1)$ & 1 \\
\hline C9 & $-0.0403(3)$ & $0.2654(4)$ & $0.4297(3)$ & $0.038(1)$ & 1 \\
\hline C10 & $-0.0359(3)$ & $0.3043(3)$ & $0.5331(3)$ & $0.030(1)$ & 1 \\
\hline C11 & $0.0931(3)$ & $0.2495(3)$ & $0.6031(3)$ & $0.028(1)$ & 1 \\
\hline C12 & $-0.1666(3)$ & $0.4024(3)$ & $0.5751(3)$ & $0.032(1)$ & 1 \\
\hline C13 & $-0.2577(3)$ & $0.3081(3)$ & $0.6779(3)$ & $0.026(1)$ & 1 \\
\hline C14 & $-0.3238(3)$ & $0.2244(3)$ & $0.6219(3)$ & $0.034(1)$ & 1 \\
\hline C15 & $-0.3770(3)$ & $0.4183(3)$ & $0.7221(3)$ & $0.040(1)$ & 1 \\
\hline C16 & $-0.1614(3)$ & $0.1934(3)$ & $0.7960(2)$ & $0.020(1)$ & 1 \\
\hline C17 & $0.1655(3)$ & $0.3732(3)$ & $0.9987(3)$ & $0.030(1)$ & 1 \\
\hline C18 & $0.2072(4)$ & $0.4040(4)$ & $0.8613(3)$ & $0.052(1)$ & 1 \\
\hline C19 & $0.2157(3)$ & $0.4545(4)$ & $1.0648(4)$ & $0.048(1)$ & 1 \\
\hline $\mathrm{H} 3 \mathrm{~A}$ & 0.4791 & -0.0118 & 0.8861 & 0.050 & 1 \\
\hline H3B & 0.5750 & -0.1084 & 0.8113 & 0.050 & 1 \\
\hline $\mathrm{H} 3 \mathrm{C}$ & 0.4939 & -0.1881 & 0.9354 & 0.050 & 1 \\
\hline $\mathrm{H} 4 \mathrm{~A}$ & 0.3808 & -0.2859 & 0.8063 & 0.056 & 1 \\
\hline $\mathrm{H} 4 \mathrm{~B}$ & 0.4586 & -0.2028 & 0.6811 & 0.056 & 1 \\
\hline $\mathrm{H} 4 \mathrm{C}$ & 0.2910 & -0.1701 & 0.6800 & 0.056 & 1 \\
\hline $\mathrm{H} 5 \mathrm{~A}$ & 0.3563 & 0.1697 & 0.6864 & 0.035 & 1 \\
\hline H5B & 0.4323 & 0.0769 & 0.6021 & 0.035 & 1 \\
\hline H7 & 0.2914 & 0.0628 & 0.4398 & 0.045 & 1 \\
\hline H8 & 0.0783 & 0.1478 & 0.3255 & 0.055 & 1 \\
\hline H9 & -0.1253 & 0.3002 & 0.3821 & 0.045 & 1 \\
\hline H11 & 0.0972 & 0.2767 & 0.6725 & 0.033 & 1 \\
\hline $\mathrm{H} 12 \mathrm{~A}$ & -0.2270 & 0.4725 & 0.4994 & 0.038 & 1 \\
\hline H12B & -0.1349 & 0.4635 & 0.6102 & 0.038 & 1 \\
\hline $\mathrm{H} 14 \mathrm{~A}$ & -0.2489 & 0.1559 & 0.5943 & 0.051 & 1 \\
\hline H14B & -0.3849 & 0.2977 & 0.5490 & 0.051 & 1 \\
\hline $\mathrm{H} 14 \mathrm{C}$ & -0.3794 & 0.1666 & 0.6869 & 0.051 & 1 \\
\hline $\mathrm{H} 15 \mathrm{~A}$ & -0.4313 & 0.3606 & 0.7888 & 0.059 & 1 \\
\hline H15B & -0.4395 & 0.4902 & 0.6498 & 0.059 & 1 \\
\hline $\mathrm{H} 15 \mathrm{C}$ & -0.3348 & 0.4728 & 0.7555 & 0.059 & 1 \\
\hline H18A & 0.2684 & 0.3114 & 0.8552 & 0.078 & 1 \\
\hline H18B & 0.2576 & 0.4817 & 0.8318 & 0.078 & 1 \\
\hline $\mathrm{H} 18 \mathrm{C}$ & 0.1225 & 0.4389 & 0.8083 & 0.078 & 1 \\
\hline H19A & 0.1824 & 0.4250 & 1.1523 & 0.072 & 1 \\
\hline H19B & 0.1783 & 0.5634 & 1.0192 & 0.072 & 1 \\
\hline
\end{tabular}




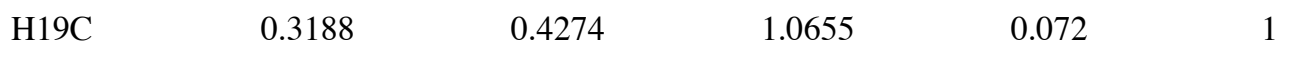

$U_{\text {eq }}$ is defined as one third of the orthogonalized $U_{\mathrm{ij}}$ tensor

Table 2a. Anisotropic Displacement Parameters

$\begin{array}{lllllll}\text { atom } & U_{11} & U_{22} & U_{33} & U_{12} & U_{13} & U_{23} \\ \text { Rh1 } & 0.017(1) & 0.016(1) & 0.020(1) & -0.008(1) & 0.000(1) & -0.006(1) \\ \text { O1 } & 0.022(1) & 0.021(1) & 0.025(1) & -0.012(1) & 0.004(1) & -0.007(1) \\ \text { O2 } & 0.021(1) & 0.020(1) & 0.024(1) & -0.007(1) & -0.004(1) & -0.006(1) \\ \text { O3 } & 0.020(1) & 0.024(1) & 0.027(1) & -0.014(1) & 0.004(1) & -0.009(1) \\ \text { O4 } & 0.024(1) & 0.020(1) & 0.021(1) & -0.008(1) & -0.003(1) & -0.008(1) \\ \text { O5 } & 0.026(1) & 0.025(1) & 0.037(1) & -0.017(1) & 0.002(1) & -0.010(1) \\ \text { C1 } & 0.018(1) & 0.025(1) & 0.017(1) & -0.006(1) & -0.001(1) & -0.004(1) \\ \text { C2 } & 0.021(2) & 0.030(1) & 0.029(2) & -0.014(1) & 0.007(1) & -0.008(1) \\ \text { C3 } & 0.022(2) & 0.038(2) & 0.037(2) & -0.015(1) & 0.004(1) & -0.005(1) \\ \text { C4 } & 0.036(2) & 0.039(2) & 0.046(2) & -0.027(2) & 0.016(2) & -0.013(1) \\ \text { C5 } & 0.028(2) & 0.037(2) & 0.029(2) & -0.016(1) & 0.009(1) & -0.017(1) \\ \text { C6 } & 0.032(2) & 0.034(2) & 0.022(2) & -0.007(1) & 0.005(1) & -0.021(1) \\ \text { C7 } & 0.035(2) & 0.054(2) & 0.032(2) & -0.022(2) & 0.007(2) & -0.019(2) \\ \text { C8 } & 0.057(2) & 0.070(2) & 0.021(2) & -0.020(2) & 0.005(2) & -0.031(2) \\ \text { C9 } & 0.040(2) & 0.052(2) & 0.021(2) & -0.005(2) & -0.008(2) & -0.022(2) \\ \text { C10 } & 0.033(2) & 0.029(1) & 0.023(2) & 0.003(1) & 0.000(1) & -0.019(1) \\ \text { C11 } & 0.033(2) & 0.028(1) & 0.022(2) & -0.004(1) & -0.001(1) & -0.017(1) \\ \text { C12 } & 0.033(2) & 0.025(1) & 0.029(2) & 0.002(1) & -0.012(1) & -0.011(1) \\ \text { C13 } & 0.023(2) & 0.026(1) & 0.027(2) & -0.005(1) & -0.005(1) & -0.008(1) \\ \text { C14 } & 0.032(2) & 0.038(2) & 0.030(2) & -0.004(1) & -0.009(1) & -0.017(1) \\ \text { C15 } & 0.030(2) & 0.035(2) & 0.044(2) & -0.011(2) & -0.011(2) & 0.002(1) \\ \text { C16 } & 0.014(1) & 0.023(1) & 0.020(2) & -0.007(1) & 0.002(1) & -0.004(1) \\ \text { C17 } & 0.021(2) & 0.020(1) & 0.048(2) & -0.015(1) & -0.006(1) & 0.001(1) \\ \text { C18 } & 0.068(3) & 0.047(2) & 0.043(2) & -0.006(2) & 0.004(2) & -0.037(2) \\ \text { C19 } & 0.032(2) & 0.043(2) & 0.087(3) & -0.043(2) & -0.001(2) & -0.011(2)\end{array}$

The general temperature factor expression: $\exp \left(-2 \mathrm{p}^{2}\left(a^{* 2} U_{11} h^{2}+b^{* 2} U_{22} k^{2}+c^{* 2} U_{33} l^{2}+2 a^{*}\right.\right.$

Table 3a. Bond Lengths (̊̊)

\begin{tabular}{|c|c|c|c|c|c|}
\hline atom & atom & distance & atom & atom & distance \\
\hline Rh1 & $\mathrm{O} 4$ & $2.0286(18)$ & $\mathrm{C} 8$ & C9 & $1.396(4)$ \\
\hline Rh1 & $\mathrm{O} 3$ & $2.0374(18)$ & $\mathrm{C} 8$ & H8 & 0.9300 \\
\hline Rh1 & $\mathrm{O} 1$ & $2.0403(18)$ & C9 & $\mathrm{C} 10$ & $1.371(4)$ \\
\hline Rh1 & $\mathrm{O} 2$ & $2.0484(18)$ & C9 & $\mathrm{C} 10$ & $1.371(4)$ \\
\hline Rh1 & O5 & $2.3042(19)$ & C9 & H9 & 0.9300 \\
\hline Rh1 & $\mathrm{Rh} 1^{1}$ & $2.3817(9)$ & $\mathrm{C} 10$ & $\mathrm{C} 11$ & $1.396(4)$ \\
\hline $\mathrm{O} 1$ & $\mathrm{C} 1$ & $1.271(3)$ & $\mathrm{C} 10$ & $\mathrm{C} 12$ & $1.520(4)$ \\
\hline $\mathrm{O} 2$ & C16 & $1.274(3)$ & $\mathrm{C} 11$ & H11 & 0.9300 \\
\hline $\mathrm{O} 3$ & $\mathrm{C} 1^{1}$ & $1.263(3)$ & $\mathrm{C} 12$ & $\mathrm{C} 13$ & $1.552(4)$ \\
\hline $\mathrm{O} 4$ & $\mathrm{C} 16^{1}$ & $1.261(3)$ & $\mathrm{C} 12$ & $\mathrm{H} 12 \mathrm{~A}$ & 0.9700 \\
\hline $\mathrm{O} 5$ & $\mathrm{C} 17$ & $1.204(3)$ & $\mathrm{C} 12$ & $\mathrm{H} 12 \mathrm{~B}$ & 0.9700 \\
\hline $\mathrm{C} 1$ & $\mathrm{O} 3^{1}$ & $1.263(3)$ & $\mathrm{C} 13$ & $\mathrm{C} 14$ & $1.517(4)$ \\
\hline $\mathrm{C} 1$ & $\mathrm{C} 2$ & $1.528(3)$ & C13 & $\mathrm{C} 16$ & $1.534(3)$ \\
\hline $\mathrm{C} 2$ & $\mathrm{C} 4$ & $1.529(4)$ & $\mathrm{C} 13$ & $\mathrm{C} 15$ & $1.537(4)$ \\
\hline
\end{tabular}




$\begin{array}{llllll}\text { C2 } & \text { C3 } & 1.535(4) & \text { C14 } & \text { H14A } & 0.9600 \\ \text { C2 } & \text { C5 } & 1.559(4) & \text { C14 } & \text { H14B } & 0.9600 \\ \text { C3 } & \text { H3A } & 0.9600 & \text { C14 } & \text { H14C } & 0.9600 \\ \text { C3 } & \text { H3B } & 0.9600 & \text { C15 } & \text { H15A } & 0.9600 \\ \text { C3 } & \text { H3C } & 0.9600 & \text { C15 } & \text { H15B } & 0.9600 \\ \text { C4 } & \text { H4A } & 0.9600 & \text { C15 } & \text { H15C } & 0.9600 \\ \text { C4 } & \text { H4B } & 0.9600 & \text { C16 } & \text { O4 }{ }^{1} & 1.261(3) \\ \text { C4 } & \text { H4C } & 0.9600 & \text { C17 } & \text { C19 } & 1.489(4) \\ \text { C5 } & \text { C6 } & 1.512(4) & \text { C17 } & \text { C18 } & 1.490(4) \\ \text { C5 } & \text { H5A } & 0.9700 & \text { C18 } & \text { H18A } & 0.9600 \\ \text { C5 } & \text { H5B } & 0.9700 & \text { C18 } & \text { H18B } & 0.9600 \\ \text { C6 } & \text { C11 } & 1.386(4) & \text { C18 } & \text { H18C } & 0.9600 \\ \text { C6 } & \text { C7 } & 1.392(4) & \text { C19 } & \text { H19A } & 0.9600 \\ \text { C7 } & \text { C8 } & 1.379(4) & \text { C19 } & \text { H19B } & 0.9600 \\ \text { C7 } & \text { H7 } & 0.9300 & \text { C19 } & \text { H19C } & 0.9600\end{array}$

Symmetry transformations used to generate equivalent atoms: ${ }^{1}-\mathrm{x},-\mathrm{y},-\mathrm{z}+2$

Table 4a. Bond Angles ( $\left(^{\circ}\right)$

\begin{tabular}{|c|c|c|c|c|c|c|c|}
\hline atom & atom & atom & angle & atom & atom & atom & angle \\
\hline $\mathrm{O} 4$ & Rh1 & $\mathrm{O} 3$ & $90.70(7)$ & $\mathrm{C} 7$ & $\mathrm{C} 8$ & H8 & 119.6 \\
\hline $\mathrm{O} 4$ & Rh1 & $\mathrm{O} 1$ & $88.69(7)$ & C9 & $\mathrm{C} 8$ & H8 & 119.6 \\
\hline $\mathrm{O} 3$ & Rh1 & $\mathrm{O} 1$ & $176.37(7)$ & $\mathrm{C} 10$ & C9 & $\mathrm{C} 8$ & $120.2(3)$ \\
\hline $\mathrm{O} 4$ & Rh1 & $\mathrm{O} 2$ & $176.38(6)$ & $\mathrm{C} 10$ & C9 & H9 & 119.9 \\
\hline $\mathrm{O} 3$ & Rh1 & $\mathrm{O} 2$ & $88.74(7)$ & $\mathrm{C} 8$ & C9 & H9 & 119.9 \\
\hline $\mathrm{O} 1$ & Rh1 & $\mathrm{O} 2$ & $91.64(8)$ & C9 & $\mathrm{C} 10$ & $\mathrm{C} 11$ & $118.5(3)$ \\
\hline $\mathrm{O} 4$ & Rh1 & $\mathrm{O} 5$ & $86.17(7)$ & C9 & $\mathrm{C} 10$ & $\mathrm{C} 12$ & $122.4(3)$ \\
\hline $\mathrm{O} 3$ & Rh1 & $\mathrm{O} 5$ & $88.80(7)$ & $\mathrm{C} 11$ & $\mathrm{C} 10$ & $\mathrm{C} 12$ & 119.1(3) \\
\hline $\mathrm{O} 1$ & Rh1 & O5 & $94.73(7)$ & C6 & $\mathrm{C} 11$ & $\mathrm{C} 10$ & $122.1(3)$ \\
\hline $\mathrm{O} 2$ & Rh1 & $\mathrm{O} 5$ & $97.39(7)$ & C6 & $\mathrm{C} 11$ & H11 & 119.0 \\
\hline $\mathrm{O} 4$ & Rh1 & $\mathrm{Rh} 1^{1}$ & $88.64(5)$ & $\mathrm{C} 10$ & $\mathrm{C} 11$ & H11 & 119.0 \\
\hline $\mathrm{O} 3$ & Rh1 & $\mathrm{Rh} 1^{1}$ & $89.00(5)$ & $\mathrm{C} 10$ & $\mathrm{C} 12$ & $\mathrm{C} 13$ & $113.8(2)$ \\
\hline $\mathrm{O} 1$ & Rh1 & $\mathrm{Rh} 1^{1}$ & $87.41(5)$ & $\mathrm{C} 10$ & $\mathrm{C} 12$ & $\mathrm{H} 12 \mathrm{~A}$ & 108.8 \\
\hline $\mathrm{O} 2$ & Rh1 & $\mathrm{Rh} 1^{1}$ & $87.78(5)$ & $\mathrm{C} 13$ & $\mathrm{C} 12$ & $\mathrm{H} 12 \mathrm{~A}$ & 108.8 \\
\hline O5 & Rh1 & $\mathrm{Rh} 1^{1}$ & $174.34(5)$ & $\mathrm{C} 10$ & $\mathrm{C} 12$ & H12B & 108.8 \\
\hline $\mathrm{C} 1$ & $\mathrm{O} 1$ & Rh1 & $119.65(16)$ & $\mathrm{C} 13$ & $\mathrm{C} 12$ & H12B & 108.8 \\
\hline C16 & $\mathrm{O} 2$ & Rh1 & 118.84(15) & $\mathrm{H} 12 \mathrm{~A}$ & $\mathrm{C} 12$ & H12B & 107.7 \\
\hline $\mathrm{C} 1^{1}$ & $\mathrm{O} 3$ & Rh1 & $118.28(16)$ & C14 & $\mathrm{C} 13$ & C16 & $110.5(2)$ \\
\hline $\mathrm{C} 16^{1}$ & $\mathrm{O} 4$ & Rh1 & $119.21(16)$ & $\mathrm{C} 14$ & $\mathrm{C} 13$ & $\mathrm{C} 15$ & $109.8(2)$ \\
\hline C17 & O5 & Rh1 & 133.7(2) & C16 & $\mathrm{C} 13$ & $\mathrm{C} 15$ & $108.1(2)$ \\
\hline $\mathrm{O} 3^{1}$ & $\mathrm{C} 1$ & $\mathrm{O} 1$ & $125.7(2)$ & C14 & $\mathrm{C} 13$ & $\mathrm{C} 12$ & $110.7(2)$ \\
\hline $\mathrm{O} 3^{1}$ & $\mathrm{C} 1$ & $\mathrm{C} 2$ & $118.4(2)$ & $\mathrm{C} 16$ & $\mathrm{C} 13$ & $\mathrm{C} 12$ & $108.9(2)$ \\
\hline $\mathrm{O} 1$ & $\mathrm{C} 1$ & $\mathrm{C} 2$ & $115.9(2)$ & C15 & $\mathrm{C} 13$ & $\mathrm{C} 12$ & $108.8(2)$ \\
\hline $\mathrm{C} 1$ & $\mathrm{C} 2$ & $\mathrm{C} 4$ & 111.2(2) & $\mathrm{C} 13$ & $\mathrm{C} 14$ & H14A & 109.5 \\
\hline $\mathrm{C} 1$ & $\mathrm{C} 2$ & $\mathrm{C} 3$ & 107.7(2) & $\mathrm{C} 13$ & $\mathrm{C} 14$ & H14B & 109.5 \\
\hline $\mathrm{C} 4$ & $\mathrm{C} 2$ & C3 & $110.0(2)$ & H14A & $\mathrm{C} 14$ & H14B & 109.5 \\
\hline $\mathrm{C} 1$ & $\mathrm{C} 2$ & C5 & 108.7(2) & $\mathrm{C} 13$ & $\mathrm{C} 14$ & $\mathrm{H} 14 \mathrm{C}$ & 109.5 \\
\hline $\mathrm{C} 4$ & $\mathrm{C} 2$ & C5 & $110.6(2)$ & H14A & $\mathrm{C} 14$ & $\mathrm{H} 14 \mathrm{C}$ & 109.5 \\
\hline C3 & $\mathrm{C} 2$ & C5 & $108.6(2)$ & H14B & $\mathrm{C} 14$ & $\mathrm{H} 14 \mathrm{C}$ & 109.5 \\
\hline $\mathrm{C} 2$ & C3 & $\mathrm{H} 3 \mathrm{~A}$ & 109.5 & $\mathrm{C} 13$ & $\mathrm{C} 15$ & H15A & 109.5 \\
\hline $\mathrm{C} 2$ & C3 & $\mathrm{H} 3 \mathrm{~B}$ & 109.5 & $\mathrm{C} 13$ & $\mathrm{C} 15$ & H15B & 109.5 \\
\hline $\mathrm{H} 3 \mathrm{~A}$ & C3 & $\mathrm{H} 3 \mathrm{~B}$ & 109.5 & H15A & C15 & H15B & 109.5 \\
\hline
\end{tabular}




$\begin{array}{llllllll}\text { C2 } & \text { C3 } & \text { H3C } & 109.5 & \text { C13 } & \text { C15 } & \text { H15C } & 109.5 \\ \text { H3A } & \text { C3 } & \text { H3C } & 109.5 & \text { H15A } & \text { C15 } & \text { H15C } & 109.5 \\ \text { H3B } & \text { C3 } & \text { H3C } & 109.5 & \text { H15B } & \text { C15 } & \text { H15C } & 109.5 \\ \text { C2 } & \text { C4 } & \text { H4A } & 109.5 & \text { O4 } 15 & \text { C16 } & \text { O2 } & 125.5(2) \\ \text { C2 } & \text { C4 } & \text { H4B } & 109.5 & \text { O4 } 1 & \text { C16 } & \text { C13 } & 117.7(2) \\ \text { H4A } & \text { C4 } & \text { H4B } & 109.5 & \text { O2 } & \text { C16 } & \text { C13 } & 116.8(2) \\ \text { C2 } & \text { C4 } & \text { H4C } & 109.5 & \text { O5 } & \text { C17 } & \text { C19 } & 121.2(3) \\ \text { H4A } & \text { C4 } & \text { H4C } & 109.5 & \text { O5 } & \text { C17 } & \text { C18 } & 121.4(3) \\ \text { H4B } & \text { C4 } & \text { H4C } & 109.5 & \text { C19 } & \text { C17 } & \text { C18 } & 117.4(3) \\ \text { C6 } & \text { C5 } & \text { C2 } & 115.2(2) & \text { C17 } & \text { C18 } & \text { H18A } & 109.5 \\ \text { C6 } & \text { C5 } & \text { H5A } & 108.5 & \text { C17 } & \text { C18 } & \text { H18B } & 109.5 \\ \text { C2 } & \text { C5 } & \text { H5A } & 108.5 & \text { H18A } & \text { C18 } & \text { H18B } & 109.5 \\ \text { C6 } & \text { C5 } & \text { H5B } & 108.5 & \text { C17 } & \text { C18 } & \text { H18C } & 109.5 \\ \text { C2 } & \text { C5 } & \text { H5B } & 108.5 & \text { H18A } & \text { C18 } & \text { H18C } & 109.5 \\ \text { H5A } & \text { C5 } & \text { H5B } & 107.5 & \text { H18B } & \text { C18 } & \text { H18C } & 109.5 \\ \text { C11 } & \text { C6 } & \text { C7 } & 118.4(3) & \text { C17 } & \text { C19 } & \text { H19A } & 109.5 \\ \text { C11 } & \text { C6 } & \text { C5 } & 119.9(3) & \text { C17 } & \text { C19 } & \text { H19B } & 109.5 \\ \text { C7 } & \text { C6 } & \text { C5 } & 121.7(3) & \text { H19A } & \text { C19 } & \text { H19B } & 109.5 \\ \text { C8 } & \text { C7 } & \text { C6 } & 119.9(3) & \text { C17 } & \text { C19 } & \text { H19C } & 109.5 \\ \text { C8 } & \text { C7 } & \text { H7 } & 120.0 & \text { H19A } & \text { C19 } & \text { H19C } & 109.5 \\ \text { C6 } & \text { C7 } & \text { H7 } & 120.0 & \text { H19B } & \text { C19 } & \text { H19C } & 109.5 \\ \text { C7 } & \text { C8 } & \text { C9 } & 120.8(3) & & & & \end{array}$

Symmetry transformations used to generate equivalent atoms: ${ }^{1}-\mathrm{x},-\mathrm{y},-\mathrm{z}+2$

Table 5a. Torsion Angles ( $\left(^{\circ}\right)$

\begin{tabular}{|c|c|c|c|c|c|c|c|c|c|}
\hline atom & atom & atom & atom & angle & atom & atom & atom & atom & angle \\
\hline $\mathrm{O} 4$ & Rh1 & $\mathrm{O} 1$ & $\mathrm{C} 1$ & 89.08(18) & $\mathrm{O} 3$ & Rh1 & $\mathrm{O} 1$ & $\mathrm{C} 1$ & $8.6(13)$ \\
\hline $\mathrm{O} 2$ & Rh1 & $\mathrm{O} 1$ & C1 & $-87.31(18)$ & O5 & Rh1 & O1 & $\mathrm{C} 1$ & $175.13(18)$ \\
\hline $\mathrm{Rh} 1^{1}$ & $\mathrm{Rh} 1$ & $\mathrm{O} 1$ & $\mathrm{C} 1$ & $0.39(17)$ & $\mathrm{O} 4$ & Rh1 & $\mathrm{O} 2$ & C16 & $-8.1(12)$ \\
\hline $\mathrm{O} 3$ & Rh1 & $\mathrm{O} 2$ & C16 & $-89.28(17)$ & $\mathrm{O} 1$ & Rh1 & $\mathrm{O} 2$ & C16 & 87.11(17) \\
\hline O5 & $\mathrm{Rh} 1$ & $\mathrm{O} 2$ & C16 & $-177.91(17)$ & $\mathrm{Rh} 1^{1}$ & $\mathrm{Rh} 1$ & $\mathrm{O} 2$ & C16 & $-0.23(17)$ \\
\hline $\mathrm{O} 4$ & Rh1 & $\mathrm{O} 3$ & $\mathrm{C} 1^{1}$ & $-89.46(18)$ & O1 & Rh1 & $\mathrm{O} 3$ & $\mathrm{C} 1^{1}$ & $-9.0(13)$ \\
\hline $\mathrm{O} 2$ & Rh1 & $\mathrm{O} 3$ & $\mathrm{C} 1^{1}$ & $86.96(18)$ & O5 & Rh1 & $\mathrm{O} 3$ & $\mathrm{C} 1^{1}$ & $-175.62(18)$ \\
\hline $\mathrm{Rh} 1^{1}$ & Rh1 & $\mathrm{O} 3$ & $\mathrm{C} 1^{1}$ & $-0.84(17)$ & $\mathrm{O} 3$ & Rh1 & $\mathrm{O} 4$ & $\mathrm{C} 16^{1}$ & 89.93(18) \\
\hline $\mathrm{O} 1$ & Rh1 & $\mathrm{O} 4$ & $\mathrm{C} 16^{1}$ & $-86.49(18)$ & $\mathrm{O} 2$ & Rh1 & $\mathrm{O} 4$ & $\mathrm{C} 16^{1}$ & $8.8(12)$ \\
\hline O5 & Rh1 & $\mathrm{O} 4$ & $\mathrm{C} 16^{1}$ & 178.67(18) & Rh1 ${ }^{1}$ & Rh1 & $\mathrm{O} 4$ & $\mathrm{C} 16^{1}$ & $0.94(17)$ \\
\hline $\mathrm{O} 4$ & Rh1 & O5 & C17 & $112.7(2)$ & $\mathrm{O} 3$ & Rh1 & O5 & C17 & $-156.5(2)$ \\
\hline $\mathrm{O} 1$ & Rh1 & O5 & $\mathrm{C} 17$ & $24.4(3)$ & $\mathrm{O} 2$ & Rh1 & O5 & C17 & $-67.9(3)$ \\
\hline $\mathrm{Rh} 1^{1}$ & Rh1 & $\mathrm{O} 5$ & C17 & $136.4(4)$ & Rh1 & $\mathrm{O} 1$ & $\mathrm{C} 1$ & $\mathrm{O} 3^{1}$ & $0.2(3)$ \\
\hline Rh1 & $\mathrm{O} 1$ & $\mathrm{C} 1$ & $\mathrm{C} 2$ & $-178.53(16)$ & $\mathrm{O} 3^{1}$ & $\mathrm{C} 1$ & $\mathrm{C} 2$ & $\mathrm{C} 4$ & $0.0(3)$ \\
\hline $\mathrm{O} 1$ & $\mathrm{C} 1$ & $\mathrm{C} 2$ & $\mathrm{C} 4$ & $178.8(2)$ & $\mathrm{O} 3^{1}$ & $\mathrm{C} 1$ & $\mathrm{C} 2$ & $\mathrm{C} 3$ & $-120.6(3)$ \\
\hline $\mathrm{O} 1$ & $\mathrm{C} 1$ & $\mathrm{C} 2$ & C3 & $58.2(3)$ & $\mathrm{O} 3^{1}$ & $\mathrm{C} 1$ & $\mathrm{C} 2$ & $\mathrm{C} 5$ & $122.0(2)$ \\
\hline $\mathrm{O} 1$ & $\mathrm{C} 1$ & $\mathrm{C} 2$ & $\mathrm{C} 5$ & $-59.2(3)$ & $\mathrm{C} 1$ & $\mathrm{C} 2$ & $\mathrm{C} 5$ & C6 & $-54.8(3)$ \\
\hline $\mathrm{C} 4$ & $\mathrm{C} 2$ & C5 & C6 & $67.6(3)$ & $\mathrm{C} 3$ & $\mathrm{C} 2$ & $\mathrm{C} 5$ & C6 & $-171.6(2)$ \\
\hline $\mathrm{C} 2$ & $\mathrm{C} 5$ & C6 & C11 & $86.7(3)$ & $\mathrm{C} 2$ & $\mathrm{C} 5$ & C6 & $\mathrm{C} 7$ & $-92.9(3)$ \\
\hline C11 & C6 & C7 & $\mathrm{C} 8$ & $-3.2(4)$ & $\mathrm{C} 5$ & C6 & C7 & $\mathrm{C} 8$ & $176.4(3)$ \\
\hline C6 & $\mathrm{C} 7$ & $\mathrm{C} 8$ & C9 & $1.9(5)$ & $\mathrm{C} 7$ & $\mathrm{C} 8$ & C9 & C10 & $-0.1(5)$ \\
\hline $\mathrm{C} 8$ & C9 & C10 & C11 & $-0.4(4)$ & C8 & C9 & C10 & C12 & $-179.4(2)$ \\
\hline C7 & C6 & C11 & C10 & $2.8(4)$ & $\mathrm{C} 5$ & C6 & C11 & C10 & $-176.8(2)$ \\
\hline C9 & C10 & C11 & C6 & $-1.0(4)$ & $\mathrm{C} 12$ & $\mathrm{C} 10$ & $\mathrm{C} 11$ & C6 & $178.0(2)$ \\
\hline C9 & C10 & C12 & $\mathrm{C} 13$ & $91.0(3)$ & $\mathrm{C} 11$ & C10 & $\mathrm{C} 12$ & C13 & $-88.0(3)$ \\
\hline
\end{tabular}




$\begin{array}{lllll}\text { C10 } & \text { C12 } & \text { C13 } & \text { C14 } & -64.9(3) \\ \text { C10 } & \text { C12 } & \text { C13 } & \text { C15 } & 174.4(2) \\ \text { Rh1 } & \text { O2 } & \text { C16 } & \text { C13 } & 179.11(16) \\ \text { C15 } & \text { C13 } & \text { C16 } & \text { O4 } 1 & 118.9(2) \\ \text { C14 } & \text { C13 } & \text { C16 } & \text { O2 } & 179.1(2) \\ \text { C12 } & \text { C13 } & \text { C16 } & \text { O2 } & 57.3(3) \\ \text { Rh1 } & \text { O5 } & \text { C17 } & \text { C18 } & 14.8(4)\end{array}$

$\begin{array}{lllll}\mathrm{C} 10 & \mathrm{C} 12 & \mathrm{C} 13 & \mathrm{C} 16 & 56.8(3) \\ \mathrm{Rh} 1 & \mathrm{O} 2 & \mathrm{C} 16 & \mathrm{O}^{1} & -0.5(3) \\ \mathrm{C} 14 & \mathrm{C} 13 & \mathrm{C} 16 & \mathrm{O}^{1} & -1.3(3) \\ \mathrm{C} 12 & \mathrm{C} 13 & \mathrm{C} 16 & \mathrm{O}^{1} & -123.0(2) \\ \mathrm{C} 15 & \mathrm{C} 13 & \mathrm{C} 16 & \mathrm{O} 2 & -60.7(3) \\ \mathrm{Rh} 1 & \text { O5 } & \mathrm{C} 17 & \mathrm{C} 19 & -165.48(19)\end{array}$

Symmetry transformations used to generate equivalent atoms: ${ }^{1}-\mathrm{x},-\mathrm{y},-\mathrm{z}+2$ 